collaborators has shown that there can be only very few antigenic determinants present in the antibody-forming cell.

The question of how antigen stimulates the potential antibody-forming cell is still a vital problem. Whether it does so by direct interaction with the reactive cell or has to go through an intermediary cell needs further clarification. Since the phagocytic cells, the macrophages, take up the antigen they have been implicated as possible intermediary cells. RNA preparations containing antigen can be extracted from macrophages and they are highly active in inducing antibody, but whether this is an essential step in the induction of antibody is not clear. Suggestions that antigen-free informational molecules are transforred from macrophages to the reactive cells have also been made, but this has not been shown convincingly. Further experimentation is required to throw light on this problem.

On the third day a discussion on allosteric enzymes was held with Dr. F. Jacob (Paris) as chairman. The fact that a combination of one molecule of ligand with a macromolecule can influence the combination of another, the same or different, has been known for a long time. The term 'allosteric' proteins was introduced by Monod and Jacob to describe proteins in which such interactions occur. Although it is to be expected that many proteins are in some degree allosteric, the introduction of this term has been especially useful in directing attention to a particularly important class of phenomena involving enzymes which, potentially at least, provide an explanation of the regulation of metabolic processes in the organism.

The original observations of such interactions were on haemoglobin which may be considered as a type case of an allosteric protein. This was the subject of Dr. Wyman's talk. Dr. Wyman discussed mainly the haem-haem interaction, considered as a model of interaction between sites for the same ligand.

The second speaker, Dr. J. Monod (Paris), described a model which aims at explaining both the interactions between similar and between different ligands, in terms of quaternary structures of proteins. In this model an allosteric protein is considered to be a polymer with an axis of symmetry, which can exist under at least two different states which are assumed to differ betwoen them by the degree of association between tho sub-units. The two states are supposed to differ in their affinity for the ligands which the protein can bind so that the presence of a given ligand can push the equilibrium towards a given state.

The third speaker was Dr. H. K. Schachman (Berkeley, California), who gave a physico-chemical description of the enzyme aspartyltranscarbamylase of $E$. coli. Dr. Schachman showed that the enzyme is made of different sub-units, some of which possess a site specific for one of the substrates, aspartate, and others a site specific for CTP which inhibits the reaction catalysed by the enzyme. Isolated sub-units still exhibit affinity for their respective ligand but without co-operative effects, those being restricted to the complex polymer.

In the discussion, many other enzymes were discussed which exhibit similar behaviour. This is the case, for example, of the enzyme dCMP amino hydrolase which has been extensively investigated by Dr. Scarano. In contrast, other complex enzymes appear to operate on a different scheme. This is the case, for example, of the enzyme glutamine synthetase of $E$. coli, investigated by Dr. E. Stadtman, an enzyme the activity of which is susceptible to partial inhibition by eight different compounds of widely different structures.

In the final session on "Molecular Aspects of Differentiation", with Prof. J. Brachet (Brussels) as chairman, the main topic discussed was the synthesis of nucleic acids and proteins during early development. Prof. Monroy (Palermo) described in detail the significance of the events following the process of fertilization in sea-urchin eggs. Ribosomes from unfertilized eggs are not capable of incorporating amino-acids, while those obtained from fertilized eggs are capable of doing so. Prof. Monroy presented the following simple experiment. When RNA from unfertilized eggs was added to liver ribosomes the latter incorporated amino-acids. But no incorporation was detected when RNA from unfertilized eggs was added to ribosomes from unfertilized eggs. He has therefore suggested that some inhibitor is present on the ribosomes which prevents them from synthesizing protein. Though the unfertilized ogg has a store of messenger RNA, the ribosomes become active only after fertilization. Prof. Monroy further indicated that inhibition on the inactive ribosomes could be lifted considerably by treating them with trypsin and removing the trypsin by washing through a sucrose layer.

Dr. D. Brown (Baltimore) examined the synthesis of ribosomal, soluble and DNA-like RNA during development of Xenopus. The kind of RNA synthesized varies conspicuously as development proceeds. Dr. Brown has been able to show that DNA-like RNA and soluble RNA are synthesized during late cleavage phase and the synthesis continues after gastrulation. The synthesis of ribosomal RNA starts only at the onset of gastrulation and increases as development proceeds.

The final paper was one on cell interactions and carcinogenesis by Dr. L. Sachs (Rehovoth), who discussed in vitro studies on the mechanism of carcinogenesis by polyoma virus and by carcinogenic hydrocarbons. In the experiments with polyoma it was shown that virus infection can induce the synthesis of cellular DNA after normal cell DNA synthesis has been repressed by contact inhibition or by X-irradiation, that each cell is induced to synthesize about double its DNA content, and that this induction is not dependent on the replication of viral DNA, but is a function of the viral genome. It was suggested that all the known experimental findings on cell-virus interactions with the small DNA tumour viruses can be explained by the synthesis of a messenger RNA early after virus infection that mediates the induction of cellular enzymes required for DNA synthesis by way of alteration of the cell surface. In the experiments with carcinogenic hydrocarbons it was shown that these chemicals can directly and rapidly induce in vitro a high frequency of transforma. tion of normal cells to tumour cells. Such in vitro investigations provide evidence on the similarities between the two types of carcinogenesis.
B. Askonas
J. A. V. ButLer
D. C. Phillips
F. $\mathrm{J}_{\mathrm{ACOB}}$
L. SACHS
G. V. Sherbet

\title{
LIVINGSTONE'S CONTRIBUTIONS TO MEDICINE
}

$\mathrm{D}$ AVID LIVINGSTONE has usually been looked on as a missionary and explorer, yet his contributions to medicine were numerous and varied. A review of them is given by $\mathrm{M}$. Gelfand in a recent issue of The Central African Journal of Medicine (11, No. 7, 192; July, 1965).

He qualified as a licentiate of the Royal Faculty of Physicians and Surgeons (Glasgow) in 1840, and throughout his life, so far as circumstances would permit, he did all ho could to keep himself fully informed and up to date. For the greater part of his time in Africa he was tolerant of native practices in medicine, seeking to understand their materials and methods. Patients flocked to him in hundreds, and by the standards of the day he was particularly successful with eye complaints and obstetrics. $\mathrm{He}$ was probably the first to describe trypanosomiasis in 
cattle, and as early as 1841 he gave arsenic to a mare infected with the disease.

When in the Tete district in 1856 the people were afflicted with a severe epidemic of smallpox, he tried to produce a vaccine by inoculating a heifer, but the inoculation failed to take; instead, he had to send to the Cape for a supply of vaccine. In the same year the Governor of Quelimane requested that he report on the causes of unhealthiness of Senna, a town on the Lower Zambesi. $\mathrm{He}$ attributed the ill-health to the low-lying nature of the land, polluted expanses of water, and decomposing vegetable matter.

Among other experiments, he carried out tests on the most suitable colours for clothing in the tropies; ho roported on a diseaso called 'maculo', now no longer' occurring in Africa, characterized by as spreading gangrene of the rectum; he described the earth-eating habits of natives in Northern Rhodesia; he wrote a vivid account of tropical ulcer among slaves; and he noticed that Africans living on a manioc diet, high in carbohydrates, suffered from disturbances of vision.

Livingstone was the first to record that the tick (Ornithodoros moubata) was the vector for relapsing fever; and he may have also been the first man of medicine to associate mosquitoes with malaria. He was aware of the great British expeditions to the Niger in 1832 and 1841, both of which ended in disastor, and after studying the account by M'William of the second expedition he came to the con- clusion that quinine was effective against malaria if it was given at an early stage and in sufficient quantity. That led him to devise his pill, consisting of quinine and purgatives, which he was able to try out in a dramatic manner on two English hunters who were dangerously ill with fever at Lake Ngami, one companion of theirs having already died. At this time his wife and children also becarne infected with malaria; he troated them success. fully in the same kind of way. It was because of this treatment that he was able to travel right across Africa from west to east in 1856 , and maintain activities in the unhealthy areas of the Zambesi and Shire Rivers for more than four years.

Livingstone also deserves credit for some attempts which he failed to carry through. When near the East Coast, for example, he camo across trees in the forests of Senna which he thought were einchonas, and he sent samples to England, only to learn from Kew that he had beon mistaken. At another time he tried to prepare chloroform for his wife when her baby was due; he had obtained some chloral hydrate, having read of Simpson's discovery, but lacked a suitable retort to prepare the anasthetic. On ib further oecasion he made use of electricity in treating a Mr. Hamilton in Bechuanaland who was suffering from paralysis.

In all these endeavours and discoveries Livingstone was moved by a pure missionary spirit, never seeking to make use of his medical knowledge for pecuniary gain.

\section{CHEMISTRY OF PLANT PIGMENTS}

A SYMPOSIUM on the chemistry of plant pigments, organized by the Phytochemical Group, was held in the University of Aberdeen on September 23-24. Attention in recent years has been given increasingly to pigments which are not readily analysed by conventional techniques either because of their lability or their intractability, and most of the substances discussed during the first day of the meeting fell into one or other of these two classes. Progress in the chemistry of the betacyanins and betaxanthins, for example, has been delayed because of their susceptibility to aerial oxidation during purification. In the first paper, Prof. M. Piattelli (Naples) described how he was able to separate these elusive nitrogenous colouring matters by fractionating plant extracts on polyamide columns. He found them occurring in plants of the Centrospermae as glycosides. Betanidin was the only aglycone of the many betacyanins, but he had discoverod as many as eight botaxanthin aglycones. These yellow pigments were found to be related in structure to betanidin and differ in that the leucodopachrome moioty of betanidin is replaced by proline, 4-hydroxyproline, glutamine, glutamic acid, aspartic acid, tyramine, dopamine, or methionine sulphoxide.

In the second lecture, Dr. F. G. Holliman (Leeds) summed up the results of several years work on the red phenazine pigments present in Pseudomonas aeruginosa. He presented convincing structural and synthetic evidence that they are 2-amino-10-methylphenazinium betaines. One of them, aeruginosin $B$, is unique as a natural product in having a sulphonic acid group on a heterocyclic system, and furthermore is only the second natural sulphonic acid to be diseovered, the first being the sulpholipid sugar moiety, sulphoquinivose. In the third paper, Prof. W. B. Whalley (London) also dealt with a Pseudomonas pigment, this time a blue pigment in $P$. lemmonieri. The compound, which analysed for CHON and the metal calcium, proved to be extremely intractable and did not respond to any of the usual physical methods of structure determination. After many attempts, Prof. Whalley eventually isolated a degradation product from chromium trioxide oxidation, a pyridone quinone with an $n$-octyl side-chain, but the full structure of the pigment remains to be elucidated.

'The afternoon session was devoted to the natural black pigments and began with an account of the melanins by Prof. R. A. Nicolaus (Naples). After discussing the chemical structure of sepiamelanin from cuttlefish ink, he went on to point out that whoroas all animal melanins examined (19 sources) were derived from 5,6-dihydroxyindole, plant molanins, such as that in Ustilago maydis spores and in nine other sources, wore derived from catechol. The question was raised, in discussion of this paper, whether plant melanins could justly be called melanins, since they did not contain nitrogen, and should instead be called humic acids. However, humic acids, as were described by Prof. R. D. Haworth (Sheffield) in the following paper, are organic pigments present in the soil, formed from decaying vegetation over the course of centuries. After a brief survey of the chemistry of humic acids, Prof. Haworth went on to outline his own recent investigations, which were based on the discovery that these compounds give electron spin resonance signals. He thought that humic acid consisted of an organic polycyclic 'core', which was responsible for electron spin resonance signals, to which metals, carbohydrates, phenolic and amino-acids are attached. He supported this view with the results from zine dust distillation of humic acids, which had produced xanthone, anthraquinone and a series of alkylnaphthalenes. Dr. H. M. Hurst (Liverpool), in a paper on the phenolic moieties in humic acid, described how he had obtained more than 30 phenolic compounds, all being lignin and flavonoid derived units, from mild reduction of humic acids with sodium amalgam in weak alkali. He suggester that humic acid was a polymer derived mainly from lignins and flavonoids, which, after microbial degradation, wero leached by rain-water from decaying leaves and deposited in the podzol. In the final paper on humic acids, Dr. R. I. Morrison (Macaulay Institute, Aberdeen) reported that, by oxidizing methylated humic acids with permanganato and identifying the products by gas chromatography, he had also obtained lignin-derived fragments. The products 